\title{
RESPONDIENDO PREGUNTAS SOBRE LA GLOBALIZACIÓN
}

\author{
DC. GUILLERMO AZNARAN CASTILLO* \\ E-mail: guillermoaznaran@hotmail.com
}

\begin{abstract}
Globalization is a controvesial historical process, is percieved in different ways, and sometimes is defined with incomprehendible concept.Thus, it's important to keep on evaluating this topic which will undoubtedly mold human lives in the upcoming centuries.
\end{abstract}

\section{RESUMEN}

La globalizacion es un proceso histórico controversial, también es percibido de formas distintas y se le define con conceptos a veces incomprensibles. Por lo tanto es pertinente seguir reflexionando sobre un tema que sin ninguna duda moldeará la vida de la humanidad en los próximos siglos.

La globalización ha merecido la atención de muchos analistas, entre políticos, economistas, intelectuales diversos, etc. quienes han estudiado este fenómeno desde diferentes enfoques, sin embargo todavía es prematuro considerar que existe una cierta teoría de la globalización; apenas si podríamos hablar de enfoques o aproximaciones contradictorias de esta realidad global.

\section{¿QUÉ ES LA GLOBALIZACIÓN?}

Es evidente que no existe unanimidad acerca del concepto y de la percepción sobre lo que es realmente el proceso llamado de globalización, el cual incorpora aspectos económicos, socioculturales, políticos, etc.; tal vez justamente esta confusión se debe a que es un fenómeno de múltiples aristas; incluso muchos autores, sobre todo europeos, prefieren nombrar el proceso como mundialización, otorgándole una acepción de simple extensión territorial.

Un aspecto importante a ser resuelto, es saber ¿a quién le conviene la globalización? Algunos podrían responder que a todos nos conviene, tanto a los países desarrollados (PD) como a los países subdesarrollados (PSD); esto podría ser cierto en un horizonte de largo plazo, porque si juzgamos por los resultados actuales, se podría concluir que el proceso de globalización ha favorecido mucho a pocos países, algo a ciertos países y nada a muchos países.

- Profesor Principal de la UNMSM, Economista graduado y doctorado en la Universidad de París, Francia, Miembro permanente del Instituto de Investigación de la Facultad de Ciencias Administrativas de la UNMSM. Profesor de Post Grado en las universidades: San Marcos, Villarreal, San Martín, Tacna. Libros publicados: Desarrollo Económico Autónomo; Cajas Rurales; Globalización: Competencias y cooperación; Crítica al Liberalismo Económico; Pequeña empresa y desarrollo regional. 
Esto sitúa al proceso de globalización en una situación de cuestionamiento que surge desde sectores sociales cada vez más numerosos, inclusive se puede afirmar que hay una coincidencia de facto entre los sectores laborales y ecologistas de los PD y aquellos marginados de los PSD por recusar el proceso de globalización. Entonces, al menos por el momento, la globalización no se presenta todavía como un proceso de mundialización del desarrollo económico-social, por lo tanto no se podría afirmar que es del interés general, ni que sea necesario que los 6000 millones de personas empujen la nave mundial hacia esa dirección.

Si aceptamos que lo arriba descrito es la situación real del proceso de globalización, es simple deducir que aquellos defensores acríticos de la globalización se refugian cada vez en mayor medida en la ideología; en efecto, los que se autotitulan "ciudadanos del mundo", o pregonan que ya existe una realidad social global o una «sociedad global», no hacen, por el momento, más que confundir sus deseos con la realidad. Es cierto que las economías y las sociedades están encaminadas hacia una mayor interrelación, pero estamos ante un proceso histórico de largo plazo que no está exento de aceleraciones, de pausas, de ritmos diferenciados entre países y de exacerbación de contradicciones sociales y nacionales.

Por si fuera poco, hay un elemento que enturbia el proceso de globalización, es como una gota de veneno en un vaso de agua pura, este elemento no es otro que la ideología del liberalismo económico, la cual pretende ser el hilo conductor del proceso de globalización, esta ideología, como es conocido, considera que en la economía y en el mercado sólo cabe la confrontación social, en otros palabras, para los liberales la competencia es la única dimensión de la economía y del mercado.

Pero como sabemos, la realidad suele ser terca y nos recuerda en cada momento que la objetividad debe estar en la base de nuestros análisis, en caso contrario caemos en la ideología justificatoria, la que busca convencernos con una retórica instrumentada sobre el común interés de los países por aceptar una globalización que se moldea a partir exclusivamente del nivel de competitividad de sus participantes.

Por eso que para U. BECK ${ }^{1}$ el actual proceso de globalización no es más que un movimiento basado en una ideología, la que no toma en cuenta los diferentes ritmos en el crecimiento económi- co de los países. Peor aún, según este autor, la ideología liberal prioriza la competitividad del mercado, convirtiéndose así en la uideología del dominio del mercado mundial». Esto es lo que podría llamarse el globalismo, entendido como una doctrina que pretende justificar toda acción, negocio o defecto, en razón que el proceso de globalización es un fenómeno lineal e ineluctable, por lo que se le debe aceptar acríticamente.

Sin ninguna duda que la ideología del globalismo presenta, en el actual momento, un status dominante; pocos analistas, empresarios, funcionarios estatales, rechazan al mercado global como el motor del proceso de globalización, es más, ellos consideran que en la medida que una población compre o venda en el mercado global está participando en la globalización. Sin embargo es justamente esta ideología del dominio del mercado el que más daño hace al proceso de globalización; pues justifica la exclusión de la mayoría, empujándola a la oposición recalcitrante.

Puestas las cosas en esta perspectiva, la globalización se presenta como un proceso contradictorio; en efecto, por un lado se presenta como una fuerza integradora, que incluye procesos productivos, regiones, naciones, etc. a través de nuevas tecnologías; pero por otro lado, se presenta como una fuerza que desintegra el mundo, pues lo divide entre ganadores y perdedores; los ganadores serían los que tienen algo que vender o comprar en el mercado global, los perdedores serían aquellos cuyos productos o ingresos cada vez son menos interesantes para el creciente mercado global. Esta perspectiva dialéctica es la que explica las grandes manifestaciones contra la globalización, de los que sienten que la globalización los excluye y les desintegra sus modos de producción y de vida.

Este escenario se podría agudizar si se mantiene la postura ideológica de considerar que la arquitectura de la globalización debería estar construyéndose únicamente a partir de los niveles de competitividad de empresas naciones e individuos; en un escenario de este tipo hay poco espacio para el desarrollo alternativo de la cooperación. No está demás repetir que la evidencia empírica nos muestra que hay individuos, naciones, regiones, procesos productivos, etc. que todavía no están en capacidad de participar en la construcción del mercado global, lo que hace imperativo explorar con creatividad formas de establecer alianzas entre las fuerzas productivas tecnológicamente atrasadas de los PSD con las empresas de los PD que ya se encuentran formando parte del mercado global. 
Este desarrollo económico alternativo, basado en la cooperación entre empresas con procesos productivos complementarios ubicados en diferentes espacios económicos, surge como una necesidad objetiva para lograr una globalización que no sea excluyente; esto tendría como resultado una globalización que sintetice los aportes individuales de cada individuo, empresa, región, nación, etc. No estaríamos asistiendo, en este caso, a una globalización de tipo neo-liberal o a una ampliación de los negocios de las grandes empresas multinacionales, sino a una globalización en tanto expresión objetiva de la comunidad de interés de los individuos organizados.

Todo esto es un confuso debate sobre lo que es realmente la globalización, determinando que al proceso se le analice sobre todo a partir de los resultados inmediatos o parciales, lo que reduce indudablemente la amplitud y la profundidad del análisis. Gran parte de lo que estamos señalando ha estado presente en el llamado Foro Internacional Globalofobia y Globalofilia. Pros y contras de la globalización ${ }^{2}$; lo que significa concretamente que deberíamos dividirnos entre los que quieren la globalización y aquellos que no la quieren.

Como si se tratara de aceptar o no la globalización, cuando lo cierto es que la globalización es un dato de la realidad, en la cual todos los países quisierán participar pero no todos son invitados, al menos no de pleno derecho; de lo que se puede concluir que existe un error cuando se plantea que frente al actual proceso de globalización caben únicamente dos alternativas: estar a favor o estar en contra de la globalización.

Por lo tanto, según esta errada postura ideológica, los que se oponen al vigente proceso de globalización no hacen más que oponerse al desarrollo histórico de la sociedad global. Con esto los opositores no serían más que unos conservaduristas de la sociedad antigua. Es necesario entonces superar esa falsa dicotomía, pues de lo que se trata no es de oponerse a la globalización sino más bien de regularla, de encaminarla, en fin, de planificarla, de tal manera que pueda convertirse en un factor de convergencia entre las sociedades, las economías y los Estados nacionales.

Para A. TOURAINE 3 , Europa está más preparada para regular la globalización, esto se debería a la fortaleza de sus instituciones y, especialmente, a su Wellfare State; sin embargo, los que se dedican a cuestionar la globalización, recusan en realidad el sistema económico capitalista; por todo esto debe señalarse que los movimientos, de por sí aislados, espontáneos e inorgánicos, en contra de la globalización, no son más que motores que empujan múltiples vehículos en diferentes direcciones, a saber, participan ecologistas, comunistas, religiosos, sindicalistas, defensores de los derechos humanos, abolicionistas de la pena de muerte, etc. Esto limita el paso de la protesta de la presentación de alternativas al tipo de globalización que tenemos en curso. Por atacar sin un programa común a la globalización, los grupos contestarios paradójicamente cuestionan todo tipo de globalización, cualquiera que sea su tipo o modalidad del proceso.

En un esfuerzo por esclarecer el carácter del proceso de globalización, A. SEN ${ }^{4}$ presenta $* 10$ verdades acerca de la globalización», veamos algunas de ellas:

Las protestas anti-globalización no son sobre globalización. Esto se refiere al carácter amplio que tienen los movimientos de protestas, contra los efectos que la globalización tiene en la economía y en la vida de las familias y de las sociedades, que no tienen el adecuado nivel de competitividad para ingresar al selectivo proceso de globalización. Pero al interior de estos movimientos ingresan reivindicaciones sociales y políticas que más bien tienen un perfil globalizante.

El asunto es si la distribución de lo ganado es justo. Éste no parece ser un punto esencial pues trata sobre la necesidad de explorar alternativas de cooperación, que están más allá del simple cálculo costo-beneficio de corto plazo. Además pone sobre la mesa el asunto referente a que en un proceso de globalización no tiene por qué existir la fatalidad ganador-perdedor.

El caso de la economía de mercado puede producir diversos resultados como sabemos el mercado es una institución necesaria pero no suficiente para que funcione una economía y una sociedad moderna. Señalamos además que la participación de las familias y las empresas en el mercado depende de la dotación de medios de producción y de la calidad del capital humano. Esto último es lo que hace la diferencia, por lo tanto, justificaría la intervención de elementos exógenos como el Estado, con el fin de equilibrar las oportunidades de ingreso al mercado o de preservar algunas actividades fuera del mercado.

La construcción global es la respuesta a las dudas globales. En este caso A. SEN considera que 
el proceso de globalización es tarea de todos, en ese sentido recomienda escuchar atentamente a los que protestan y a los que dudan, porque ambos sectores sociales forman parte del proceso. Lo cierto es que a nadie le conviene que las protestas y las dudas vayan en ascenso, porque podría llegarse, reiteramos, al cuestionamiento mismo de cualquier proceso de globalización, cualquiera que sea el tipo, características o sus efectos, de lo que resultaría el renacimiento del localismo o nacionalismo antihistórico.

\section{¿POR QUÉ SE PRODUCE LA GLOBALIZACIÓN?}

Deberíamos comenzar por preguntarnos ¿cuándo se inició el proceso de globalización? Al respecto surgen muchas respuestas, todas coincidentemente toman como epicentro a Europa Occidental; una respuesta podría ser el viaje de Marco Polo a la lejana china, otra el viaje de Cristóbal Colón a América, o los viajes de piratas y comerciantes marítimos por todos los océanos. Podría tomarse también como punto de partida las expansiones de los imperios Romano, Español, lnglés, los cuales si bien pertenecen a épocas distintas, tienen un denominador común: extender los negocios a los rincones más alejados de la tierra, utilizando para fin cualquier medio, de la zanahoria al garrote.

No sería exagerado afirmar que en tanto proceso la globalización siempre ha estado presente en la historia económica mundial, cada nueva conquista militar, cada nuevo convoy comercial, cada nueva inversión en ultramar, cada nuevo préstamo externo, han significado un paso adelante hacia el mítico mercado único, el que se constituiría en base para contar con una sociedad única, moldeada de acuerdo a los intereses de aquellos que dirigían el proceso de globalización; en consecuencia si quisiéramos contestar la pregunta ipor qué se produce la globalización?, estamos en la necesidad de hacer un análisis histórico. Veamos:

En primer lugar, el surgimiento del modo de producción capitalista ha significado la aparición de la competencia por la obtención de utilidades por encima del promedio social; este afán de lucro no se detuvo en las fronteras de los nacientes Estados nacionales, al contrario los negocios para expandirse fuera de las fronteras tuvieron que apoyarse en la fuerza político-militar de su Estado nacional. Resultando así una dupla victoriosa: Estado-Empresa Multinacional (EM).

En segundo lugar, el Estado nacional tiene un rol importante en el proceso de globalización, pues si bien los Estados actuales no tienen el rol predominante de las épocas en que los negocios en ultramar necesitaban del apoyo político-militar del Estado, igualmente en la actualidad los Estados garantizan las condiciones sociales y políticas para que funcionen los negocios; recordemos que la ampliación de los negocios fuera de las fronteras ha necesitado la presencia activa del Estado, quien a través de organismos internacionales, acuerdos entre países o intervenciones militares, ha defendido los intereses de sus EM.

Sin embargo hay que precisar que actualmente los Estados desempeñan un rol secundario, con mucho activismo en la formación del capital humano, pero que han reducido enormemente su acción directa en el campo económico; le corresponde ahora su acción directa en el campo económico; le corresponde ahora a las EM conducir el proceso de globalización, en efecto son las EM las encargadas de movilizar los recursos financieros para una inversión en cualquier parte del mundo, son también las EM los que desarrollan y transfieren tecnología; por esa razón si se quiere analizar seriamente el proceso histórico que ha tenido la globalización, tenemos que partir del análisis del desarrollo de las empresas capitalistas, y saber por qué estas EM se han desplazado a todas las zonas del mundo, orientando sus negocios con una estrategia que comprende un escenario cada vez más amplificado.

Entonces si queremos analizar el proceso histórico de la globalización, tenemos que empezar analizando cómo la empresa capitalista del siglo $\mathrm{XIX}$ se ha transformado en lo que ahora conocemos como empresa multinacional (EM); evidenciando que las respuestas a la problemática del crecimiento de las EM se convierta en la respuesta a la problemática de la globalizacón; esta identificación entre las problemáticas del crecimiento de las EM y de la globalización, es tal vez la principal característica del actual proceso de globalización, así como es la característica que en definitiva diferencia a esta etapa histórica de las otras, en las cuales también hubo avances hacia el mercado global, pero lo que faltaba era el extraordinario desarrollo de las fuerzas productivas del siglo XX.

Por un lado, tenemos que el desarrollo de las fuerzas productivas ha sido el resultado de la aplicación de los nuevos conocimientos científicos y tecnológicos a los procesos productivos y a las decisiones gerenciales. Por otro lado, la matriz ciencia-tecnología-producción-gestión, ha significado un crecimiento exponencial de las utilidades empresariales, basados en lo que K. Marx Ila- 
mó la "plusvalía relativa». Estas utilidades empresariales generadas en los negocios de las EM, han necesitado ser rentabilizadas en forma ampliada, es decir que se han requerido nuevos y mayores negocios; los que de acuerdo a las nuevas tecnologías empresariales podrían estar localizadas de manera integral o fraccionada en cualquier parte del mundo. Si hacemos una agregación de los puntos geográficos que comprenden esta localización de los negocios, entonces nos encontramos con lo que se podría denominar el mercado global, base objetiva del actual proceso de globalización.

Entonces se puede intentar una síntesis, lo primero fue el desarrollo de la ciencia y de la tecnología, después estos nuevos conocimientos se aplicaron a los procesos productivos y de gestión, esto incrementó las utilidades empresariales, las que tuvieron que buscar nuevas oportunidades de rentabilización. Por lo tanto la globalización se explica en gran medida por las necesidades que tienen las EM de ampliar la esfera de sus negocios. Como nunca en la historia las empresas pueden producir suficientes bienes y servicios para toda la humanidad, pero esa producción tiene que ser rentable, es decir las EM deben encontrar lugares en el mundo en donde los negocios sean rentables.

Por otro lado hay que tener en cuenta que el desarrollo de las telecomunicaciones y la informática han permitido generar nuevos negocios, pero lo más importante es que aquel desarrollo permite que los procesos de producción puedan ser localizados en cualquier parte del mundo. En otras palabras está desapareciendo la necesidad de ubicar todo el proceso productivo en un solo lugar. Además, también está desapareciendo la ubicación coincidente entre centro de producción y mercado.

Esta extensión del escenario de acción de las EM, ha determinado una intensificación de la competencia, obligando que las EM busquen asegurar sus propias zonas de influencia; estas zonas no son entendidas solamente como mercados sino también como potenciales aliados que participan en alguna de las fases del proceso productivo. De esta manera se va construyendo lo que se denomina la estrategia global.

Otro factor importante que empuja el proceso de globalización paradójicamente son los problemas fácticos de carácter global que existen a nivel de todo el mundo. Tales como el fundamentalismo en varias regiones del mundo, incluyendo sus versiones terroristas, asimismo tenemos el tráfico de drogas generalizado en todos los países, el deterioro del medio ambiente (la capa de ozono, los cambios climáticos, la deforestación, etc.), la persistencia de una serie de conflictos bélicos de focalización regional (Medio Oriente, los Balcanes, África, etc.). Todo esto amerita una coordinación política supraestatal.

Gran parte de estos temas fueron tratados en el aludido Seminario en Madrid ${ }^{5}$, en el cual se plantearon algunas opiniones muy interesantes acerca de las implicancias políticas del proceso de globalización, al respecto se pueden señalar lo manifestado por S. SASSEN sobre la presunta desaparición del Estado frente a estructuras mayores; el concepto de una estructura suprarracional aparece entonces como una necesidad. Es más, personajes como N. SERRA señalaron que «Europa se tiene que convertir en un motor mundial para regular la globalización». Por su parte L. JOSPIN añadió que «la globalización es algo que hay que aprender a construir y gobernar».

De lo que resulta que actualmente el proceso de globalización también es una necesidad política, pues es la única manera de ejecutar soluciones que tienen que ver con la viabilidad política, económica, social, ambiental, etc. Por lo tanto a la hora actual hay razones de política suprarracional para avanzar en el proceso de globalización. Finalmente, tenemos las opiniones de G. SOROS "Vivimos en una economía global, pero la organización política de nuestra sociedad global es deplorablemente insuficiente. Nos vemos privados de la capacidad de mantener la paz y contrarrestar los excesos de los mercados financieros. Sin estos controles, es probable que la economía global se desplome».

Es clara la posición de G. SOROS sobre la necesidad que el proceso de globalización se encamine hacia la creación de una organización política, además, piensa, que esto sería beneficioso para el funcionamiento de los mercados financieros, de por sí ya bastante inestables. Con más precisión G. SOROS recomienda que "para estabilizar y regular una economía verdaderamente global, es necesario algún sistema global de toma de decisiones políticas». Esta posición es compartida por S. AMIN ${ }^{7}$ pero desde una visión política distinta, al respecto este autor propone "un proyecto humanista y alternativo de globalización, compatible con una perspectiva socialista. La realización de ese proyecto implica construir un sistema político global». En conclusión, existe una relación complementaria entre la globalización y un Estado suprarracional; de un lado el proceso 
de globalización necesita un marco jurídico-político suprarracional para consolidarse; por otro lado, un Estado para poder convertirse en una realidad con jurisdicción suprarracional necesita que el proceso complete su tarea de desintegrar los Estados nacionales.

Entonces hay razones económicas y políticas para que se desarrolle, se consolide $y$, por último, sea irreversible el proceso de globalización; resumiendo podemos señalar algunas de estas razones: el avance de la informática y de las telecomunicaciones, la creciente competitividad en los negocios que determina la ampliación del escenario de disputa, la estandarización del sistema de necesidades, lo que uniforma la demanda en términos globales, y, por supuesto, la universalidad de ciertos problemas que necesitan ser enfrentados con políticas y organismos de carácter global.

\section{Notas}

1 U. BECK (1998), p. 17.

2 Seminario: Globalofobia y globalofilia: Pros y contras de la globalización. Curso de verano de la Universidad Complutense, Madrid, 2001.

3 A. TOURAINE (2001), p. 1.

4 A. SEN (2001), p. 1-2.

5 Seminario Globalofilia y Globalofobia; Saskia Sassen es profesora en la Universidad de Chicago (USA); Narcia Serra es un ex Presidente del gobierno español. Lionel Jospin es el Primer Ministro del gobierno francés.

6 GEORGE SOROS (1999), p. 21, 31, 32.

7 SAMIR AMIN (1999), p. 19 